\title{
APLICAÇÕES ELETROANALÍTICAS COM ELETRODOS DE PRATA CONFECCIONADOS A PARTIR DE CDS GRAVÁVEIS
}

Eduardo M. Richter, Dosil P. de Jesus, Carlos A. Neves, Claudimir L. do Lago e Lúcio Angnes*

Departamento de Química Fundamental, Instituto de Química, Universidade de São Paulo, Av. Prof. Lineu Prestes, 748, 05508900 São Paulo - SP

Recebido em 13/12/02; aceito em 29/4/03

\begin{abstract}
ELECTROANALYTICAL APPLICATIONS OF SILVER ELECTRODES BUILT FROM RECORDABLE CDs. This paper presents a new way for the construction of silver electrodes utilizing recordable CDs. The new electrodes were explored for the quantification of chloride (by potentiometry/FIA), cyanide (by amperometry/FIA) and for lead analysis (by square wave voltammetry). For the flowing measurements, a digital multimeter, connected to a microcomputer (via RS 232 interface) was used to collect directly the potential signal (for $\mathrm{Cl}^{-}$measurements) or to take the output signal from a potentiostat (for $\mathrm{CN}^{-}$measurements). The square wave voltammetry analysis was performed in a commercial instrument. The results shown good performance of the new electrodes and the detection limit $(\mathrm{s} / \mathrm{n}=3)$ attained for these three analytes were: $0.2,50$, and $200 \mu \mathrm{g} \mathrm{L}^{-1}$ for $\mathrm{Pb}^{2+}, \mathrm{Cl}^{-}$, and $\mathrm{CN}^{-}$respectively.
\end{abstract}

Keywords: silver CD-R electrodes; interfaced multimeter; electroanalysis.

\section{INTRODUÇÃO}

Durante muitos anos, mercúrio se constituiu no material mais empregado como eletrodo em aplicações eletroanalíticas. No entanto, a alta toxicidade deste metal e a estreita faixa de trabalho na região anódica fizeram com que os eletrodos sólidos passassem a ganhar crescente importância. Dentre os eletrodos sólidos, receberam mais atenção os eletrodos de carbono (vítreo, pirolítico e de pasta), ouro, platina e prata. Além destes, em menor escala, também são utilizados eletrodos de cobre, níquel, alumínio, antimônio, paládio, molibdênio, entre outros ${ }^{1}$. Uma das principais vantagens do eletrodo de mercúrio é a possibilidade da sua renovação através da troca da gota, principalmente em meios onde ocorre o envenenamento do eletrodo. Eletrodos sólidos não apresentam esta vantagem e para a renovação de sua superfície diferentes procedimentos de limpeza são descritos ${ }^{2-4}$

Uma condição intermediária pode ser alcançada com eletrodos descartáveis. Trata-se de dispositivo de baixo custo, de construção simples e rápida, que pode ser descartado sempre que apresentar algum problema e substituído por outro. Os eletrodos impressos constituem-se em exemplo bastante popular de eletrodos descartáveis ${ }^{5}$. Recentemente foi descrito um processo de construção de eletrodos de ouro de baixo custo, que permite descartar o sensor sempre que for necessário ${ }^{6}$. Tais eletrodos foram construídos a partir de discos compactos graváveis (CD-Rs). Devido à simplicidade de construção e ao baixo custo dos CD-Rs, estes eletrodos podem ser utilizados e, posteriormente, descartados em casos de envenenamento, formação de óxidos sobre sua superfície ou ainda algum outro problema.

CD-Rs possuem em sua constituição uma película metálica de ouro ou prata. Os eletrodos de ouro já foram explorados em nosso grupo para a quantificação de traços de metais pesados ${ }^{7,8}$ e para a análise de fármacos ${ }^{9,10}$. A construção de eletrodos de prata a partir de CD-Rs também é de grande relevância. Eletrodos de prata convencionais são muito utilizados para medidas potenciométricas e sua aplicação em análises amperométricas e voltamétricas tem recebido crescente atenção. Potenciometricamente, vários analitos foram

*e-mail: luangnes@iq.usp.br quantificados, dentre os quais, haletos ${ }^{11-14}$, cianeto $^{15}$, vitamina $\mathrm{C}$ e glutationa ${ }^{16}$. Amperometricamente, cianeto, sulfeto, iodeto, brometo e formaldeído foram analisados por diferentes autores ${ }^{17-19}$. Por voltametria, sulfato ${ }^{20}$, zinco ${ }^{21}$, nitrato ${ }^{22}$ e $\mathrm{DNA}^{23}$ também foram quantificados utilizando eletrodos de prata. Em medidas de redissolução ("stripping"), estes eletrodos foram empregados para análise de diversas espécies químicas, dentre as quais: chumbo ${ }^{24-26}$, cádmio $^{24}$, arsenato $^{27}$, tiofosfamida ${ }^{28}$ e sulfito ${ }^{29}$. Eletrodos de prata também foram modificados, através da imobilização de tio-compostos para determinação de mioglobina ${ }^{30}$ e com histidina para quantificação de $\mathrm{NAD}^{31}$.

Neste trabalho é descrita uma forma simples de obtenção de eletrodos de prata a partir de CD-Rs (CDtrodos). Suas potencialidades foram avaliadas frente a vários analitos, onde foram obtidos resultados semelhantes àqueles alcançados utilizando eletrodos de prata comerciais. Além do baixo custo representado pelos eletrodos de prata desenvolvidos (a partir de um CD podem ser feitas dezenas de eletrodos), um outro aspecto a ser destacado neste estudo foi a utilização de um multímetro dotado de interface RS 232 e software para aquisição de dados, que pode ser utilizado nas medidas em fluxo. Para a análise de cloreto, as medidas potenciométricas puderam ser feitas diretamente pelo multímetro, enquanto que para a detecção amperométrica em fluxo, o sinal foi adquirido diretamente da saída de um potenciostato. Esse multímetro permite interfacear qualquer sistema de detecção, cuja resposta seja em potencial, corrente ou resistência, em função do tempo. Os dados obtidos podem facilmente ser transferidos para a maioria dos programas de tratamento de dados.

\section{PARTE EXPERIMENTAL}

\section{Composição dos discos compactos graváveis (CD-Rs)}

CD-Rs são geralmente constituídos por quatro ou cinco camadas sobrepostas de diferentes materiais. Esses dispositivos possuem uma base de policarbonato, sobre a qual é depositada uma fina camada de um polímero fotodegradável (ftalocianinas, cianinas ou azocompostos) que são alterados no processo de gravação. Sobre essa camada polimérica é depositada, por "sputtering", uma fina pe- 
lícula metálica (de ouro ou prata), a qual geralmente possui uma espessura de 50 a $100 \mathrm{~nm}$ e área total da ordem de $100 \mathrm{~cm}^{2}$. Essa última camada é protegida por um ou dois filmes poliméricos. $\mathrm{O}$ ponto chave para confecção de eletrodos a partir de CD-Rs é encontrar uma forma prática de expor o filme metálico.

\section{Construção dos eletrodos de prata a partir dos CD-Rs}

Para a obtenção de eletrodos metálicos a partir de CD-Rs faz-se necessário o acesso à camada metálica existente nos mesmos. Em CD-Rs de ouro, essa tarefa é facilitada, uma vez que as películas de proteção podem ser facilmente removidas pela ação de ácido nítrico concentrado ${ }^{6}$. No caso de CD-Rs de prata, esse processo não pode ser utilizado, uma vez que o ácido concentrado acaba dissolvendo a camada metálica. Com a finalidade de substituir o ácido nítrico foram testados diferentes solventes orgânicos como clorofórmio, acetona, benzeno, tolueno, e vários outros. Entretanto, os resultados não foram satisfatórios devido à remoção apenas parcial das camadas protetoras ou contaminação da película de prata. Um processo alternativo e viável foi desenvolvido para ter acesso à camada de prata, como é mostrado na Figura 1 . Na etapa a, uma lâmina de policarbonato recebe uma fina camada de adesivo epóxi (Araldite Brascola Ltda - Brasil) e é posicionada sobre as camadas de proteção da película de prata (lado oposto ao que ocorre gravação ou leitura do CD-R). O tamanho dessa lâmina determina a área do filme de prata removido. Após a secagem, com o auxílio de uma lâmina de corte (estilete) as películas de proteção e o filme metálico são cortados, contornando a lâmina de policarbonato. Em seguida, a lâmina de policarbonato é destacada trazendo consigo a película de prata de mesma área da lâmina utilizada (etapa b da Figura 1). Para utilização em células estacionárias, a lâmina colada sobre o CD-R é pequena, tipicamente $3,0 \mathrm{~cm}$ x $0,5 \mathrm{~cm}$ e a área do eletrodo é definida com o auxílio de uma resina (esmalte de unhas). O contato elétrico ${ }^{8}$ é feito utilizando um fio comum pressionado sobre a película de prata com o auxílio de uma fita de Teflon ${ }^{\circledR}$.
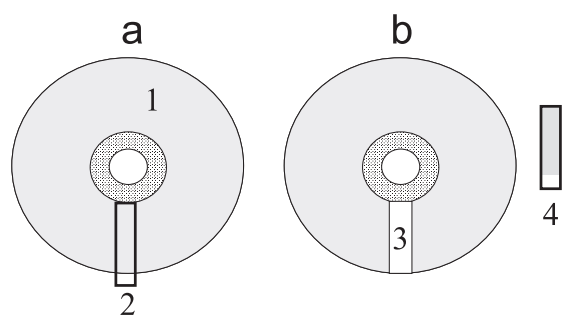

Figura 1. Construção do CDtrodo de prata: 1- CD-R de prata; 2- lâmina de policarbonato; 3- região do CD-R onde a prata foi removida; 4- lâmina de policarbonato com o filme de prata

\section{Célula para análises em fluxo}

A célula em fluxo, representada na Figura 2, é composta por dois blocos de acrílico $(4,5 \times 2,5 \times 2,8 \mathrm{~cm})$, um eletrodo de prata com dimensões de 1,5 x 3,0 cm (construído de forma similar ao descrito na seção anterior) e um espaçador flexível de polietileno, silicone ou borracha. $\mathrm{O}$ conjunto é prensado por dois parafusos. $\mathrm{O}$ orifício central do espaçador determina a área efetiva do eletrodo de prata e sua espessura define o volume total da célula.

Neste sistema em fluxo, o transporte das soluções é feito através de tubos de polietileno (1 mm de diâmetro interno), sendo os orifícios dos blocos de acrílico do mesmo diâmetro. O contato elétrico no eletrodo de trabalho pode ser feito por conexão direta da garra "jacaré" do equipamento ou através de um fio comum fixado à extre- midade do mesmo. O eletrodo de referência ( $\mathrm{Ag} / \mathrm{AgCl}, \mathrm{NaCl}$ sat) foi construído no próprio laboratório ${ }^{32}$ e um tubo de aço inoxidável (posicionado na saída da célula) atuou como eletrodo auxiliar. Nesta célula (Figura 2), não há a necessidade de fixar (de forma hermética) o eletrodo de referência, uma vez que o duto de descarte (i) se localiza em um nível inferior ao da altura máxima do tubo cônico (h) onde o eletrodo de referência é posicionado.

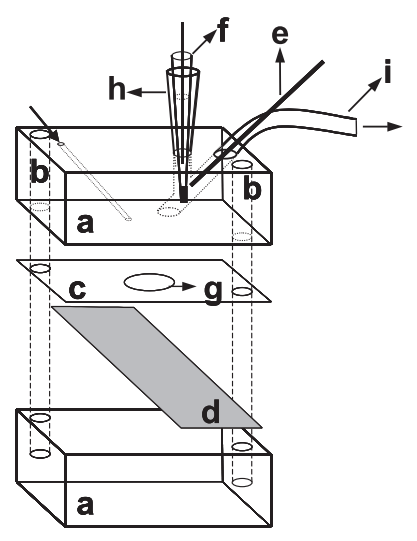

Figura 2. Célula FIA: (a) blocos de acrílico; (b) furos dos parafusos; (c) espaçador (silicone); (d) fatia de CD-R de prata; (e) eletrodo auxiliar (tubo de aço); (f) eletrodo de referência $\mathrm{Ag} / \mathrm{AgCl}, \mathrm{NaCl}$ sat; ( $g$ ) área do eletrodo (definida pelo espaçador); ( $h$ ) ponteira de micropipeta com a ponta cortada; (i) tubo de polietileno

\section{Pré-tratamento do CDtrodo}

Após a obtenção do CDtrodo de prata, faz-se necessária uma limpeza prévia do mesmo, pois parte do material fotodegradável permanece sobre a superfície metálica quando esta é deslocada do CDR. A utilização da ciclovoltametria mostrou-se rápida e eficaz para este propósito. A realização de 30 ciclos consecutivos de $-300 \mathrm{mV}$ a $+450 \mathrm{mV}$ com uma velocidade de varredura de potencial de $200 \mathrm{mV} / \mathrm{s}$ em meio de $\mathrm{NaNO}_{3} 0,1 \mathrm{~mol} \mathrm{~L}^{-1}$ e de $\mathrm{HNO}_{3} 0,01 \mathrm{~mol} \mathrm{~L}^{-1}$ é suficiente para obter resultados muito satisfatórios. Nos primeiros ciclos (Figura 3a), o sinal varia significativamente, porém, nos últimos o sinal apresenta-se bastante estável (Figura 3b). Após esta etapa o eletrodo pode ser utilizado para medidas potenciométricas ou voltamétricas.

\section{Reagentes e soluções}

Todas as soluções foram preparadas utilizando água deionizada do sistema de purificação NANOpure de Barnstead $\left(18 \mathrm{M} \Omega \mathrm{cm}^{-1}\right)$. Os reagentes utilizados $\left(\mathrm{NaCl}, \mathrm{NaCN}, \mathrm{NaOH}, \mathrm{NaNO}_{3}, \mathrm{HNO}_{3}\right.$ ) foram todos de grau analítico (Merck). O eletrólito utilizado para as análises de cloreto e de chumbo foi uma solução em $\mathrm{NaNO}_{3} 0,1 \mathrm{~mol} \mathrm{~L}^{-1}$ e de $\mathrm{HNO}_{3} 0,01 \mathrm{~mol} \mathrm{~L}^{-1}$. Para análise de cianeto, o eletrólito utilizado foi uma solução de $\mathrm{NaOH} 0,1 \mathrm{~mol} \mathrm{~L}^{-1}$. As soluções padrão foram preparadas por diluição de soluções estoque, preparadas a partir dos sais mencionados anteriormente, exceto para o chumbo, para o qual foi utilizada uma solução estoque de $1000 \mathrm{mg} \mathrm{L}^{-1}$ comercial (Merck).

\section{Instrumentação}

Os experimentos de voltametria de redissolução por onda quadrada foram realizados em um potenciostato PGSTAT 20 Autolab (Eco Chemie B.V. - Utrecht, Netherlands) interfaceado a um microcomputador. O software (GPES) deste equipamento foi utilizado no tratamento dos dados. 

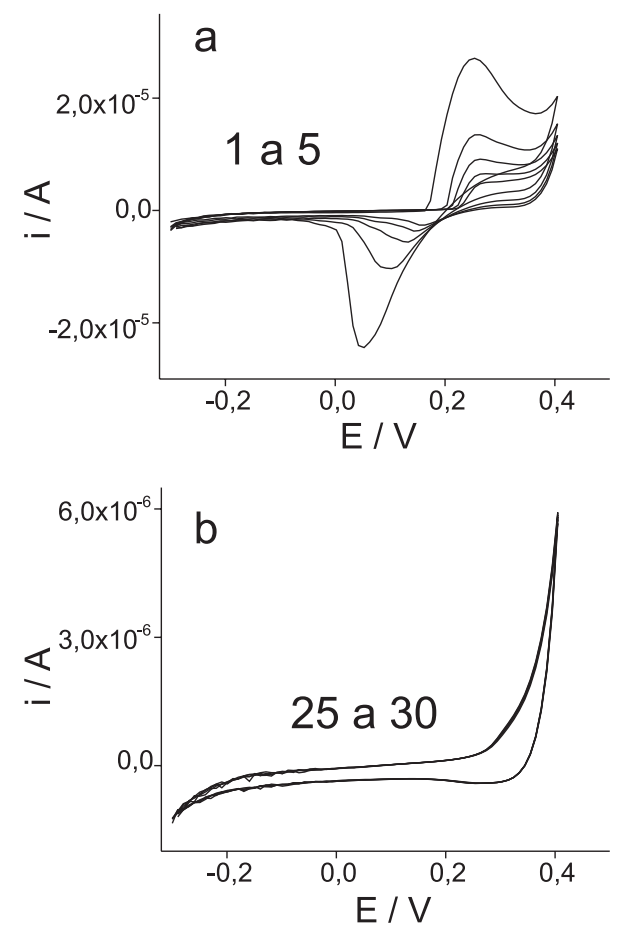

Figura 3. Pré-tratamento do CDtrodo. (a) 5 primeiros ciclovoltamogramas; (b) 5 últimos ciclovoltamogramas; eletrólito: de $\mathrm{NaNO}_{3} 0,1 \mathrm{~mol} \mathrm{~L}^{-1}$ e de $\mathrm{HNO}_{3} 0,01 \mathrm{~mol} \mathrm{~L}^{-1}$; velocidade de varredura de potencial: $200 \mathrm{mV} / \mathrm{s}$; faixa de varredura: $-300 \mathrm{mV} \mathrm{a}+450 \mathrm{mV}$

Para as medidas potenciométricas e amperométricas foi utilizado um sistema FIA, cujo esquema é mostrado na Figura 4. O bombeamento da solução transportadora é realizado através de um mini-compressor de ar do tipo bomba de diafragma, originalmente destinado ao borbulhamento de ar em aquários domésticos ${ }^{33}$. O controle da vazão da solução é feito pelo ajuste de duas válvulas (minitorneiras, utilizadas para regular o fluxo de ar em aquários). A forma de controlar o fluxo pode ser entendida com o auxílio da Figura 4. Via de regra, a torneira (d) é mantida completamente aberta, enquanto que o grau de abertura da segunda torneira (c) determina a pressão que vai ser exercida sobre a solução e, conseqüentemente, determina o fluxo do eletrólito, contido no frasco (e). Havendo necessidade de fluxos muito baixos, o fechamento parcial da torneira (d) facilita o ajuste em (c). As soluções padrão e as amostras foram injetadas com o auxílio de um injetor-comutador manual (CENA - Piracicaba Brasil).

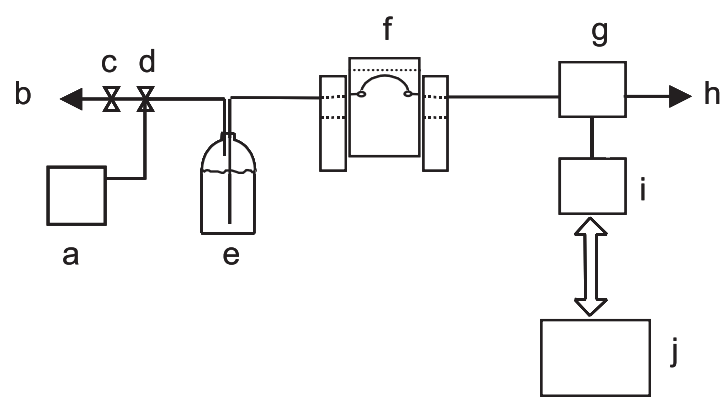

Figura 4. Sistema FIA: (a) mini-compressor de ar; (b) saída do ar excedente; (c,d) válvulas de controle de vazão (2 e 3 vias, respectivamente); (e) reservatório da solução transportadora; $(f)$ injetor comutador-manual; $(g)$ célula FIA; (h) descarte; (i) multímetro; (j) microcomputador
Para as medidas de potencial foi utilizado um multímetro modelo HC 608 (Hung Chung- Seoul, Korea), interfaceado a um microcomputador. Esse multímetro possui uma interface RS232 e é acompanhado de um software (Protec 608-versão 1.0) para aquisição de dados a uma taxa máxima de 2 medidas por segundo. Os dados são armazenados em um arquivo texto, compatível com a maioria dos programas de tratamento de dados. Foi utilizado um circuito seguidor de tensão (amplificador operacional OPA 606 - Burr Brown), devido à alta impedância oferecida pela célula eletroquímica, nas medidas potenciométricas para cloreto. Um filtro RC, com constante de tempo de $130 \mu$ s, foi utilizado para filtrar os ruídos.

Para as medidas amperométricas foi utilizado um potenciostato modelo 400 (EG\&G - USA). Este potenciostato foi interfaceado ao computador através da leitura do potencial de saída (destinado para o registrador) com o multímetro descrito anteriormente. Os dados destes experimentos foram tratados utilizando o software Microcal Origin 5.0.

\section{RESULTADOS E DISCUSSÃO}

Para avaliar o desempenho dos CDtrodos de prata confeccionados a partir de CD-Rs, vários sistemas conhecidos para este tipo de eletrodo foram testados. Como sensor potenciométrico, sua resposta foi avaliada para íons cloreto. No sistema amperométrico, seu comportamento foi estudado para análise de cianeto. Como sensor voltamétrico, os eletrodos foram avaliados para a detecção de íons $\mathrm{Pb}^{2+}$. Além da avaliação dos CDtrodos de prata, foi avaliado o desempenho do multímetro para aquisição dos dados via computador nos experimentos potenciométricos e amperométricos em fluxo.

\section{Medidas potenciométricas}

A Figura 5a mostra um fiagrama obtido para concentrações crescentes de cloreto na faixa de 5 a $50 \mathrm{mg} \mathrm{L}^{-1}$. Esta resposta é linear em função do logaritmo da concentração de cloreto, $(R=0,998 ; n=6)$, concordando com o previsto pela equação de Nernst. Apesar de se tratar de um sistema de injeção em fluxo, a vazão utilizada $(2 \mathrm{~mL} /$ min) e a faixa de concentração permitem que o sistema atinja o equilíbrio ${ }^{13}$. Para esta condição, é possível uma frequiência analítica de 75 determinações $\mathrm{h}^{-1}$. A Figura $5 \mathrm{~b}$ apresenta um fiagrama para concentrações decrescentes de cloreto na faixa de 4 a $0,25 \mathrm{mg} \mathrm{L}^{-1}\left(\mathrm{R}^{2}=\right.$ 0,$998 ; \mathrm{n}=6$ ) e dois sinais em triplicata para duas amostras comerciais de água mineral ( $\mathrm{g}$ e h). Neste caso, como as concentrações de $\mathrm{Cl}$ são relativamente baixas, o comportamento do sistema não é linear em função do logaritmo da concentração, se for mantida a mesma vazão do experimento apresentado na Figura 5a. Este fato, a primeira vista, parece paradoxal, visto que uma das vantagens dos métodos em fluxo é permitir que se trabalhe em condições em que não se atinge o equilíbrio termodinâmico. No entanto, é necessário lembrar que a equação de Nernst só é válida para sistemas em equilíbrio. Para baixas concentrações, a equação de Nernst só é obedecida se forem utilizadas vazões baixas, de forma a permitir que seja atingido o equilíbrio nestas condições. Uma forma alternativa de se trabalhar nesta faixa de concentração é adotar um ajuste de segunda ordem entre a variação de potencial $(\Delta \mathrm{E})$ e a concentração do analito. Este processo foi repetido várias vezes, obtendo-se resultados altamente reprodutíveis. Trabalhando-se desta forma, foi possível usar uma vazão de $7 \mathrm{~mL} / \mathrm{min}$, aumentando a frequiência analítica para 240 determinações $\mathrm{h}^{-1} \mathrm{e}$ possibilitando determinar a concentração de cloreto em águas minerais comerciais sem a necessidade de pré-concentração da amostra. A concentração de cloreto calculada para a amostra $\boldsymbol{h}$ foi $2,11 \pm 0,02 \mathrm{mg} \mathrm{L}^{-1}$. O conteúdo de cloreto na amostra $\boldsymbol{g}$ é inferior à menor concentração dos padrões injetados e sua concentração foi estimada em $0,12 \pm 0,01 \mathrm{mg} \mathrm{L}^{-1}$. 

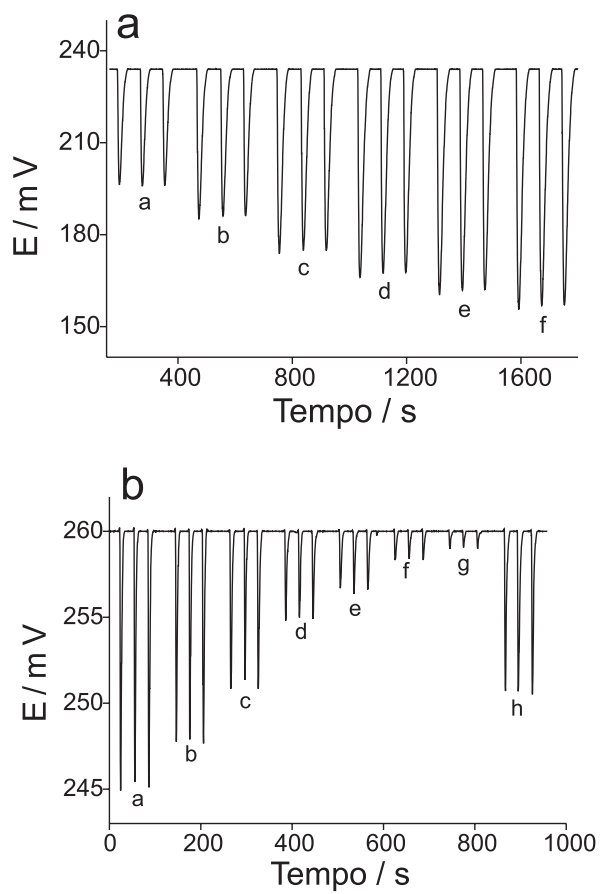

Figura 5. Fiagramas potenciométricos para concentrações crescentes (a) $e$ decrescentes (b) e seus respectivos gráficos em função do logaritmo da concentração e versus concentração de $\mathrm{Cl}$ : (a): (a) 5; (b) 10; (c) 20; (d) 30; (e) 40; (f) $50 \mathrm{mg} \mathrm{L}{ }^{-1}$; vazão: $2 \mathrm{~mL} / \mathrm{min}$. (b): (a) 4; (b) 3; (c) 2; (d) 1; (e) 0,5; (f) $0,25 \mathrm{mg} \mathrm{L}^{-1} ;(\mathrm{g}, h)$ amostras de água mineral. Vazão: $7 \mathrm{~mL} / \mathrm{min}$. Eletrólito: $\mathrm{NaNO}_{3} 0,1 \mathrm{~mol} \mathrm{~L}^{-1}$ e $\mathrm{HNO}_{3} 0,01 \mathrm{~mol} \mathrm{~L}^{-1}$; volume injetado: $130 \mu \mathrm{L}$

\section{Medidas amperométricas}

Para as medidas amperométricas também foi utilizada a célula FIA apresentada na Figura 2 e um CDtrodo de prata, sob um potencial fixo aplicado de $0 \mathrm{~V}$ em relação a um eletrodo de referência de $\mathrm{Ag} / \mathrm{AgCl}, \mathrm{NaCl}$ sat. Como o potenciostato da EG\&G modelo 400 converte a corrente medida em um potencial correspondente, esses dados foram adquiridos acoplando-se o multímetro diretamente na saída destinada ao registrador. Dessa forma, os dados puderam ser facilmente transferidos para um microcomputador e analisados num programa de tratamento de dados.

Na Figura 6 são apresentados os sinais obtidos em injeções sucessivas de concentrações crescentes de cianeto (2,1 a 10,4 mg L $\left.\mathrm{L}^{-1}\right)$. Observou-se uma excelente linearidade $(\mathrm{R}=0,99995 ; \mathrm{n}=5)$ o que demonstra que o CDtrodo de prata apresenta um comportamento comparável a um eletrodo de prata comercial descrito na literatura.

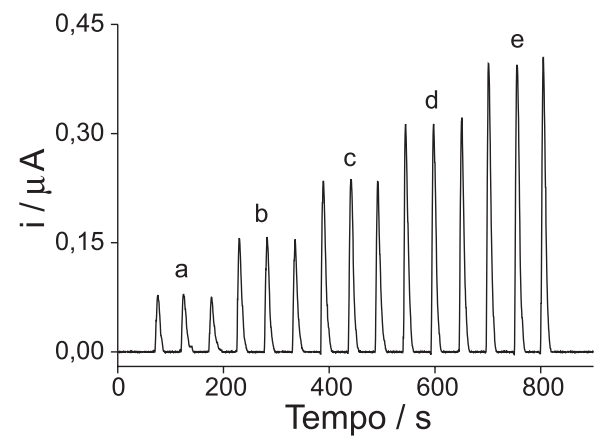

Figura 6. Amperograma para injeções crescentes de $C N$. (a) 2,1; (b) 4,1; (c) 6,2; (d) 8,3; (e) 10,4 $\mathrm{mg} \mathrm{L}^{-1}$. Eletrólito: $\mathrm{NaOH} \mathrm{0,1} \mathrm{mol} \mathrm{L}^{-1}$; potencial: $0 \mathrm{~V}$; volume injetado: $130 \mu \mathrm{L}$; vazão: $3 \mathrm{~mL} / \mathrm{min}$

\section{Medidas voltamétricas}

Na Figura 7a são apresentados os sinais obtidos por redissolução por onda quadrada para concentrações crescentes de $\mathrm{Pb}^{2+}$ na faixa 1,4 a $18 \mu \mathrm{g} \mathrm{L}^{-1}$. A regressão linear utilizando a área dos picos resultou num coeficiente de correlação de 0,999 . Neste experimento foi utilizado um potencial de pré-tratamento antes de cada análise de $-100 \mathrm{mV}$ durante $20 \mathrm{~s}$, potencial de depósito de $-700 \mathrm{mV}$ e um tempo de deposição de $180 \mathrm{~s}$. A redissolução foi realizada variandose o potencial de $-550 \mathrm{mV}$ a $0 \mathrm{~V}$ com uma freqüência de $20 \mathrm{~Hz}$. $\mathrm{Na}$ Figura $7 \mathrm{~b}$ nota-se a repetibilidade de resposta para $10 \mu \mathrm{g} \mathrm{L}^{-1} \mathrm{~Pb}^{2+} \mathrm{em}$ 20 medidas sucessivas, onde o desvio padrão relativo calculado foi de apenas de $2,5 \%$.

O coeficiente de correlação muito próximo da unidade e o baixo desvio relativo confirmam o bom desempenho dos CDtrodos de prata para a análise de chumbo, na faixa de $\mu \mathrm{g} \mathrm{L}^{-1}$ ou mesmo em concentrações inferiores, usando tempos de depósitos maiores. Estes experimentos foram realizados em uma célula estacionária com sistema de três eletrodos: eletrodo de trabalho (CDtrodo), auxiliar (platina) e referência $(\mathrm{Ag} / \mathrm{AgCl}, \mathrm{NaCl}$ sat).
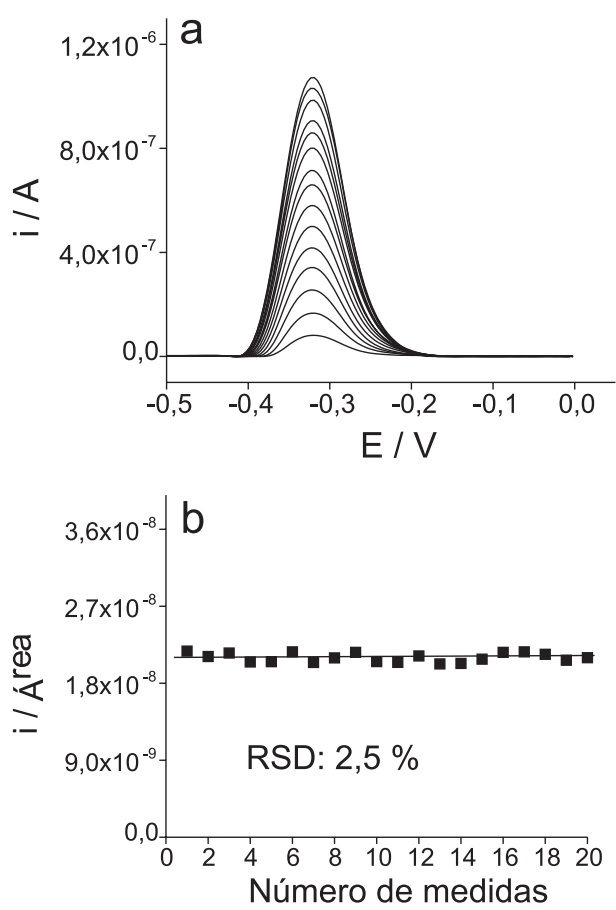

Figura 7. (a) Voltamogramas de onda quadrada para concentrações crescentes de $\mathrm{Pb}^{2+}\left(1,4\right.$ a $\left.18 \mu \mathrm{g} \mathrm{L}^{-1}\right)$; (b) estudo da repetibilidade de sinal para $10 \mu \mathrm{g} \mathrm{L}^{-1} \mathrm{~Pb}^{2+}$ com desvio padrão relativo de 2,5\% $(n=20)$. Condições: potencial de depósito: $-700 \mathrm{mV}$; tempo de depósito: $180 \mathrm{~s}$; freqüência: 20 $\mathrm{Hz}$; eletrólito: de $\mathrm{NaNO}_{3} 0,1 \mathrm{~mol} \mathrm{~L}^{-1}$ e de $\mathrm{HNO}_{3} 0,01 \mathrm{~mol} \mathrm{~L}^{-1}$

\section{CONCLUSÕES}

Os eletrodos de prata confeccionados a partir de CD-Rs apresentam várias características: facilidade de construção, baixo custo e versatilidade, permitindo sua utilização associada a células em fluxo e estacionárias. Tais propriedades possibilitam a construção de vários eletrodos a partir de um CD-R (o número de unidades depende da área dos eletrodos construídos) o que permite que os mesmos sejam utilizados como eletrodos descartáveis. Essa característica é muito importante, de acordo com o meio em que o eletrodo é utilizado. Os resultados obtidos para medidas potenciométricas, ampero- 
métricas e voltamétricas demonstram que as características dos CDtrodos de prata são similares aos eletrodos de prata comerciais.

Aliado à simplicidade e baixo custo do sistema de detecção descrito neste trabalho, o uso do sistema de aquisição de dados empregando um multímetro interfaceável (acompanhado do software de aquisição de dados) apresentou excelentes resultados. Após a instalação do software, este multímetro serve para a aquisição de sinais em sistemas cuja resposta seja em potencial, corrente ou resistência. A taxa máxima de aquisição de dados de 2 medidas por segundo é muito satisfatória para as técnicas utilizadas neste estudo, nas quais os eventos ocorrem na escala de alguns segundos ou mais. A combinação dos CDtrodos, sistema de aquisição de dados, célula FIA e o uso de mini-compressores de ar para impulsionar as soluções no sistema FIA torna todo o arranjo experimental extremamente simples e de custo reduzido, porém, sem perda na qualidade dos resultados.

\section{AGRADECIMENTOS}

Os autores agradecem à FAPESP, ao CNPq (projeto RENAMI) e à CAPES pelo suporte financeiro e pelas bolsas de pesquisa e produtividade.

\section{REFERÊNCIAS}

1. Richter, E. M.; Dissertação de Mestrado, Universidade de São Paulo, Brasil, 2000; http://www.teses.usp.br/teses/disponiveis/46/46133/tde21032003-094629/

2. Yang, Z.; Gonzalezcortes, A.; Jourquin, G.; Vire J. C.; Kauffmann, J. M.; Delplancke, J. L.; Biosens. Bioelectron. 1995, 10, 789.

3. Poon, M.; Mccreery, R. L.; Anal. Chem. 1987, 59, 1615.

4. Brett, C. M. A.; Angnes, L.; Liess, H. D.; Electroanalysis 2001, 13, 765.
5. Nascimento, V. B.; Angnes, L.; Quim. Nova 1998, 21, 614.

6. Richter, E. M.; Augelli, M. A.; Kume, G. H.; Mioshi, R. N.; Angnes, L.; Fresenius J. Anal. Chem. 2000, 366, 444.

7. Angnes, L.; Richter, E. M.; Augelli, M. A.; Kume, G. H.; Anal. Chem. 2000, 72,5503 .

8. Richter, E. M.; Augelli, M. A.; Magarotto, S.; Angnes, L.; Electroanalysis 2001, 13, 760 .

9. Muñoz, R. A. A.; Matos, R. C.; Angnes, L.; J. Pharm. Sci. 2001, 90, 1972.

10. Muñoz, R. A. A.; Matos, R. C.; Angnes, L.; Talanta 2001, 55, 855.

11. Alegret, S.; Alonso, J.; Bartroli, J.; Garciaraurich, J.; Martinezfabregas, E.; J. Pharm. Biomed. Anal. 1988, 6, 749 .

12. Seefeld, S.; Baltensperger, U.; Anal. Chim. Acta 1993, 283, 246.

13. Chen, Z.; Hibbert, D. B.; Anal. Chim. Acta 1997, 350, 1.

14. Below, H.; Kahlert, H.; Fresenius J. Anal. Chem. 2001, 371, 431.

15. Frenzel, W.; Liu, C. Y.; Oleksy-Frenzel, J.; Anal. Chim. Acta 1990, 233, 77.

16. Kolar, M.; Dobcnik, D.; Radic, N.; Pharmazie 2000, 55, 913.

17. Rocklin, R. D.; Johnson, E. L.; Anal. Chem. 1983, 55, 4.

18. Pihlar, B.; Kosta, L.; Anal. Chim. Acta 1980, 114, 275.

19. Shi, Y. L.; Johnson, B. J.; Analyst 1996, 121, 1507.

20. Skvortsova, L. I.; Kiryushov, V. N.; J. Anal. Chem. 2002, 57, 255.

21. Kizek, R.; Sevcikova, S.; Smarda, J.; Jelen, F.; Anal. Biochem. 2002, 301,

22. Krista, J.; Kopanica, M.; Novotny, L.; Electroanalysis 2000, 12, 199.

23. Fan, C. H.; Song, H. Y.; Li, X. F.; Anal. Biochem. 1999, 271, 1.

24. Bonfil, Y.; Brand, M.; Kirowa-Eisner, E.; Anal. Chim. Acta 2002, 464, 99.

25. Zen, J. M.; Yang, C. C.; Kumar, A. S.; Anal. Chim. Acta 2002, 464, 229.

26. Brand, M.; Eshkenazi, I.; Kirova-Eisner, E.; Anal. Chem. 1997, 69, 4660.

27. Chemezova, K. S.; J. Anal. Chem. 2001, 56, 384.

28. Zakharova, O. M.; Zakharov, M. S.; J. Anal. Chem. 2001, 56, 1044.

29. Skvortsova, L. I.; Kiryushov, V. N.; Tarasova, V. A.; J. Anal. Chem. 1999, $54,562$.

30. Long, Y. T.; Shu, J. J.; Chen, H. Y.; Fresenius J. Anal. Chem. 1998, 360, 614.

31. Long, Y. T.; Zou, J.; Chen, H. Y.; Anal. Lett. 1997, 30, 2691.

32. Pedrotti, J. J.; Angnes, L.; Gutz, I. G. R.; Electroanalysis 1996, 8, 673.

33. Matos, R. C.; Gutz, I. G. R.; Angnes, L.; Fontenele, R. S.; Pedrotti, J. J.; Quim. Nova 2001, 24, 795 . 